Results Since the project began it has:

- consulted with staff, parents and young people to better inform practical implementation of the Standards,

- developed an internal online and face to face training program which has been rolled out to more than 2,263 internal stakeholders,

- developed and implemented a centralised reporting and investigation procedure managed by an Internal Response Team,

- developed Council's Child Safety Policy;

- drafted a Child Safe Procedural Guide to operationalise Council's commitment to keep children and young people safe.

Conclusions Child safeguarding is now an integral part of our organisational strategy.

Council has transparent governance arrangements in place including accessible child safeguarding policies and procedures, a code of conduct, a risk management framework, and clear accountabilities for staff of all levels.

In addition, Casey is committed to continuous improvement and learning from incidents, identified risks and ongoing activities that engage children and young people with Council and in their safety and wellbeing.

\section{A.003 POTENTIAL CRASH-RISKS FOR VEHICLES IN STAGGERED CAR-FOLLOWING ON MULTILANE-HIGHWAYS UNDER LOW-DENSITY CONDITIONS}

Kar Pranab*, PadinjareVenthuruthiyil Suvin, Chunchu Mallikarjuna. Indian Institute of Technology, Guwahati, India

\subsection{6/injuryprev-2021-safety.35}

The challenges associated with evaluating the traffic flow quality on multilane highways are manifold. Better quality of driving is achieved when mobility and safety are optimally tradedoff. The majority of the studies on Level of Service (LOS) have primarily focused on the improvement of travel time, or travel speed by minimizing the travel delay. However, the safety aspect/risk-taking behavior of drivers remains ignored. Rear-end crashes are the most frequently occurring one that accounts for about $29 \%$ of all the crashes and resulting in a significant number of injuries and fatalities each year. The presence of slow-moving vehicles in highspeed highways would lead to car-following situations even in lower density conditions, which impart potential crash risks. The current study evaluates the risky behavior of drivers by identifying the potential conflict between the leading and lagging vehicles. The study performs both macroscopic and microscopic diagnosis with the help of detailed trajectory as well as detector data. We analyzed the variability in both speed and acceleration characteristics of drivers corresponding to a potential conflict scenario. The conflict points were identified by using the surrogate safety measures termed Time-to-Collision (TTC) and Stopping-Sight-Distance (SSD). Results indicate that even under low-density conditions, the potential risk of rear-end crash is high due to the presence of significant slow-moving vehicles. The acceleration characteristics indicate that many drivers accept potentially risky maneuvers under the presence of slowmoving vehicle ahead. The crash potential due to such a situation could be minimized by regulating the designated lanes for slow-moving vehicles.

\section{A.004 BUILD CONFIDENCE AMONG POLICY-DECISION MAKERS BY REMOVING CONCERNS FOR CSR LAW IMPLEMENTATION}

Cui MinYan(Monica)*, Chunyan(Amidy) Peng* . Safe Kids China, Shanghai, China

10.1136/injuryprev-2021-safety.36

Background No national law mandating child restraint systems (CRS) is in China. Shanghai passed traffic rules in 2017 requiring the use of CRS, but with no penalties. Implementation is weak due to significant government concerns. Using research and marketing data, Safe Kids China (SKC) developed targeted activities to remove the concerns of the policymakers to promote CRS implementation.

Objective Implementation of the law on CRS in Shanghai traffic rules.

Method SKC allayed concerns among decisionmakers by: 1) Highlighting the importance of CRS law, sharing global experience, and providing technical support to stakeholders via seminars; 2) Presenting parents' support for CRS and data on increasing CRS purchasing trends, and assisting new parents' CRS usage at hospitals; 3) Training certified child passenger safety technicians (CPST) involving police and healthcare staff. Results Shanghai decision-makers submitted a policy proposal to strengthen CRS implementation. The related government agencies include CRS education into their work. Police have included CRS into their training. The police coordinated other agencies' resources for the public education. Five policemen were trained as child passenger safety techinician (CPST) and 12 policemen received CRS training. The CDC distributed posters to 1600 kindergartens. Police conduct CRS checks. However, penalties are not to be issued for CRS violations.

Conclusion In March 2017, Shanghai issued the amended traffic rules, including a provision that children under 4 riding in a family car must use CRS. However, continuous promotion and political buy-in is required to have more strict rules on CRS.

\section{B - Drowning - Factors, March 23, 2021}

\section{A.001 FATAL DROWNING IN WHO REGION OF THE AMERICA'S - A LEADING KILLER}

Stephen Beerman*. 'University of British Columbia, Faculty of Medicine, Dept of Family Practice, Nanaimo, Canada; ${ }^{2}$ Canadian Drowning Prevention Coalition, Fredericton, Canada

\subsection{6/injuryprev-2021-safety.37}

Background Drowning is a serious, preventable and neglected public health issue resulting an estimated 295,210 death per year, worldwide. An estimated 1 Billion people live in the WHO Region of the Americas/Pan American Health Organization (PAHO) in 53 countries and territories. There is an estimated 23,096 drowning deaths per year based on GBD 2017 data.

The PAHO works with countries to improve and protect health. PAHO engages in technical cooperation with member countries. The PAHO does not have a program for Drowning Prevention.

Methods Using PAHO website Leading Causes of Death data, to focus on the ranking of drowning as a leading causes of death. The last 5 years of available data in all age groups, were reviewed 
Results The PAHO website includes all age group fatal drowning data for $45 / 53$ countries and territories. Drowning is a top 5 cause of all deaths in $42 / 45$ countries and territories, in at least one age group. Drowning is the lead cause of death in 15/45 nations, in at least one age group. The greatest burden of fatal drowning is children and youth. Fatal drowning rates are greatest in LMICs.

Conclusions Fatal Drowning is a significant public health issue in the WHO Region of the Americas/Pan American Health Organization. Drowning Prevention strategies should become priority public health interventions in all countries and territories, with a focus on children and youth.

Learning The WHO Region of the Americas/PAHO should develop a program for drowning prevention focused on proven interventions for high burden countries.

\section{B.002 SERIOUSLY GUYS? YOUNG MALES AND COASTAL DROWNING DEATHS IN AUSTRALIA}

Jaz Lawes*, Luke Strasiotto, Shane Daw. Surf Life Saving Australia, Sydney, Australia

\subsection{6/injuryprev-2021-safety.38}

Males are consistently overrepresented in fatal coastal drowning incidents, with young males representing a significant proportion of these. This is often attributed to their greater participation and increased confidence levels which do not necessarily reflect abilities.

Using annual coastal safety surveys and the national coastal fatality database, this study examined characteristics of drowning deaths and current perceptions of coastal safety within this high-risk demographic. Inflated confidence levels of young males to engage in risk-taking behaviour was confirmed with survey respondents consistently considering themselves to be experienced enough to take risks during activities in comparison to the total adult population.

Since 2004, males aged between 16-39 account for $36 \%$ of all drowning deaths. In 2018-19, 44 drowning deaths were young males. Average fatality rate ratios revealed young males are twice as likely $(2.05: 1)$ to drown on our coast, particularly at rock/cliff and beach locations. Young males were more likely to drown while jumping, snorkelling, swimming and wading, with $58 \%$ of young male drowning victims reported to have little to no experience in the activity being undertaken at the time of death. Analyses revealed young males were twice as likely to drown on a public holiday and significantly more likely to have consumed alcohol or drugs $(\mathrm{RR}=1.3$; CI:1.1-1.5) than other demographics.

These results highlight that the adventurous nature and overconfidence exhibited by young males puts them at significant risk of drowning and should be a target for future drowning prevention strategies on the Australian coast.

\section{B.003 WHAT MAKES A POPULATION 'HIGH-RISK' FOR DROWNING IN AUSTRALIA?}

\footnotetext{
1,2 Stacey Willcox-Pidgeon* ${ }^{*}{ }^{1,2}$ Richard Franklin, ${ }^{2}$ Peter Leggat, ${ }^{2}$ Sue Devine, ${ }^{1}$ Justin Scarr. ${ }^{1}$ Royal Life Saving Society - Australia, Sydney, Australia; ${ }^{2}$ James Cook University, Townsville, Australia
}

10.1136/injuryprev-2021-safety.39
Background In Australia, people born overseas are considered at higher-risk of drowning. Common perceptions is that being from overseas (including tourists), with English as another language makes someone more vulnerable to drowning. The aims were to analyse drowning deaths of people born overseas, and to identify specific risk factors to better inform prevention strategies targeted to migrant populations.

Methods A total population analysis of all unintentional, drowning deaths over 10 years (2008/09-2017/18) of people born outside of Australia were included. Cases were extracted from the Royal Life Saving National Fatal Drowning Database, collated from the National Coronial Information System. Descriptive statistics, relative risk and crude drowning rates were calculated.

Results 794 people drowned, who were born overseas, accounting for $27 \%$ of total drowning deaths. $77 \%$ were men, 24\% were aged 25-34 years. Three groups were identified: Australian residents (83\%), overseas visitors (14\%) and international students (4\%). Differences in drowning rates were found when analysed by country of birth, and time in Australia e.g. new arrivals $0-5$ years versus resident for 5-10 years, 10-20 years and 20 years + .

Conclusion These results identified that distinct sub-groups exist within the 'high-risk' population category, each with differing risk factors for drowning. These findings offer practical insights when devising effective drowning prevention policy and programs for specific groups. This study informed the Australian Water Safety Strategy 2030 area pertaining to 'highrisk' populations.

Learning Outcomes 'High-risk' populations for drowning are not homogenous and this should be taken into account when developing drowning prevention policies and strategies

\section{B.004 ANOTHER GENDER DATA GAP: WOMENS DROWNING IN NEW ZEALAND}

Karen Wells*. Water Safety New Zealand, Wellington, New Zealand

\subsection{6/injuryprev-2021-safety.40}

Background There is currently a paucity of literature regarding female drowning worldwide. The majority of research on drowning discusses female drowning as a secondary topic to male drowning. Drowning fatalities are predominantly males in New Zealand which has potentially led to a lack of focus on female drowning. This research is the first of its kind in New Zealand.

Method This research first outlines the breadth and consequences of the gender data gap. Analysis of DrownBase (New Zealand's drowning data base) data from the last 40 years has been undertaken. This data analysis focused on trends in female drowning data over the last 40 years to identify the risk factors relating to female drowning and how they have changed.

Results Results show that the issue of female fatal and nonfatal drowning is a growing problem. Female drownings are rising in New Zealand, with 20\% more fatalities and a $30 \%$ increase in non-fatal drownings in 2018 compared to the 2013-2017 five-year average. Female drowning differs to male drowning, in $201861 \%$ of female fatalities were a result of accidental immersions (in contrast to $35 \%$ of male fatalities). 